\title{
Prevalence of cryptococcosis among HIV-infected patients in Yaounde, Cameroon
}

\author{
*Dzoyem JP', Kechia FA², Ngaba GP³ , Lunga PK¹, Lohoue $\mathrm{PJ}^{2}$
}

1. Faculty of Science, University of Dschang, Cameroon.

2. Faculty of Medicine and Biomedical Sciences, University of Yaoundé I, Cameroon.

3.Faculty of Medicine and Pharmaceutical Sciences, University of Douala, Cameroon

\begin{abstract}
Background: Cryptococcus neoformans is encapsulated yeast which causes life-threatening infections in up to $40 \%$ of AIDS patients in Africa.

Objective: To investigate the prevalence of cryptococcosis among HIV infected patients in Yaounde.

Methods: In a hospital-based surveillance study of cryptococcosis, the colonization of Cerebrospinal Fluid (CSF), urine and blood sample by $\mathrm{C}$. neoformans was evaluated by direct microscopic examination and culture techniques. Data obtained were then analyzed based on the medical records of the patients.

Results: Among the105 patients sampled for the study, the CD4 counts varied between 31 and 304 lymphocytes $/ \mathrm{mm}^{3}$. Direct specimens examination $(n=294)$ in India ink preparations revealed polysaccharide capsule in $25(8.5 \%)$ of the samples. Upon culture, 29 (9.86 \%) samples were positive of C. neoformans (23 from the CSFs and 6 from the urine). All the positive samples were obtained from patients who were not on Antiretroviral Therapy (ART). Meningo-encephalitis symptoms were observed in 13 patients with C. neoformans in CSFs.

Conclusion: This study reveals that cryptococcosis is rife in AIDS patients in Yaounde. Therefore, to minimize the death toll, we recommend that its routine check should be integrated in the management of HIV/AIDS patients.

Key words: Cryptococcosis, HIV/AIDS patients, prevalence, Yaounde.

African Health Sciences 2012; 12(2): 129-133 http://dx.doi.org/10.4314/ahs.v12i2.8
\end{abstract}

\section{Introduction}

The HIV pandemic has greatly contributed to the emergence of opportunistic fungal pathogens and increased incidence of infections over the last two decades. Cryptococcosis is a cosmopolitan but rare opportunistic mycosis, caused by an encapsulated yeast Cryptococcus neoformans. This fungus causes lifethreatening infections in approximately $7-15 \%$ of patients with HIV/AIDS around the world, and up to $40 \%$ in Africa $^{1}$. In 1993, the United States Centers for Disease Control and Prevention (CDC) reported that $6 \%$ of the 274150 patients with AIDS developed cryptococcal disease in the United States. Furthermore, patients with AIDS-associated cryptococcal infections now account for $80 \%-90 \%$ of all patients with cryptococcosis ${ }^{2}$. In immunocompromised patients infection with $C$. neoformans is often fatal. Patients usually require life-

\begin{tabular}{|l|}
\hline *Correspondence author: \\
Dr. Jean Paul Dzoyem \\
Faculty of Science \\
University of Dschang \\
Cameroon \\
Tel: +23799245686 \\
E.mail: jpdzoyem@,yahoo.fr \\
\hline
\end{tabular}

African Health Sciences Vol 12 No 2 June 2012 long suppressive therapy. The overall mortality rate from cryptococcal meningitis is approximately $25 \%$ $30 \%$. Of those who survive, $40 \%$ have significant neurological disorders, including loss of vision, decreased mental function, hydrocephalus, and cranial nerve palsies, with relapse occurring in up to a quarter of cases ${ }^{3}$. Although cryptococcosis is considered an opportunistic infection particularly in AIDS patients, the data on prevalence vary in time and space. This may be as a result of the changing epidemiology of HIV infection and the AIDS management pattern ${ }^{4}$. In sub-Saharan Africa with the highest burden of HIV/AIDS, incidence and mortality of cryptococcosis are yet to be well documented. In this study we report the prevalence of Cryptococcus neoformans in three specimen types (CSF, urine and blood) obtained from HIV-infected patients at the Yaoundé Central Hospital, Cameroon.

\section{Methods \\ Patients and samples}

A hospital-based surveillance of cryptococcosis was conducted at the Yaounde Central Hospital (the major reference centre for HIV/AIDS management in Cameroon) from March 2010 to December 2010. 
Patients that met our condition i.e. evidence in the medical records of HIV seropositivity were eligible. For each included patient, the following basic data were collected: age and gender; CD4 counts at admission; antiretroviral therapy (ART) regimen and antifungal therapy. Ethical clearance and permission to perform record reviews and patient interviews after informed consent was given by the Faculty of Medicine and Biomedical Sciences (FMBS), Yaounde-Cameroon, and the Yaounde Central Hospital (YCH) Ethical committees.

\section{Laboratory methods}

The CSFs, urine, and blood samples were aseptically collected in sterile tubes, and transfer to the Mycology laboratory for immediate processing. The diagnostic tests for cryptococcosis used in this study included India ink microscopic observation and agar cell cultures. Each sample was centrifuged at 3000 $\mathrm{rpm}$ for $10 \mathrm{~min}$. One drop of the pellet (urine and CSF) or serum was used for the India ink preparation. The remaining pellet or $0.1 \mathrm{ml}$ serum was spread on agar slants in three tubes each of Sabouraud-chloramphenicol medium and incubated at $30^{\circ} \mathrm{C}$ for up to 3 weeks. Isolates were subcultured in Sabouraud dextrose agar slants in screw-top glass test tubes to confirm purity. C. neoformans was identified on the basis of colony morphology and by subculturing isolates onto Niger seed agar (NSA) and urease agar. Growth at $37^{\circ} \mathrm{C}$ with brown discoloration on NSA and urease hydrolysis identified the genus. Furthermore, doubtful isolates or isolates with undetermined reactions were subjected to API 20C Aux (BioRad). Clinical history was recorded for all the cases diagnosed of cryptococcosis. Clinical manifestations recorded were neurological manifestations (headache, mental confusion, vomiting, and virtual alterations), cutaneous manifestations (skin lesions), respiratory manifestations (cough), and general manifestations (fever, weight loss).

\section{Results}

The105 HIV-infected patients recruited for this study were made up of 65 females and 40 males giving a sex ratio of 1.6. Their characteristics are shown in table 1.

Table 1: Characteristics of the population studied

\begin{tabular}{lcllll}
\hline & \multicolumn{1}{c}{ CDC classification categories Total } \\
\cline { 2 - 4 } & A & B & C & \\
\hline Number of patients & 10 & 35 & 60 & 105 \\
Age range (years) & $23-55$ & $17-45$ & $21-43$ & $17-55$ \\
Sex M $\quad$ M & 6 & 17 & 22 & 40 \\
& F & 4 & 18 & 38 & 65 \\
Antiretroviral treatment & 3 & 14 & 32 & 49 \\
Positive C. neoformans & 0 & 8 & 21 & 29 \\
\hline
\end{tabular}

The ages of patients were between 17 and 55 years. Out of 294 samples processed (97 CSF, 105 urine and 92 serums), 21 yielded growth of C. neoformans, giving a prevalence of $7.14 \%$. The results of the analyzed samples are shown in table 2 .

Table 2: Results of samples analysis

\begin{tabular}{lccccc}
\hline & \multicolumn{3}{c}{ Type of sample } & Total & $\%$ \\
\cline { 2 - 4 } & CSF & Urine & Blood & & \\
\hline Number collected & 97 & 105 & 92 & 294 & \\
Positive India Ink & 20 & 5 & 0 & 25 & $8.5 \%$ \\
Positive culture & 23 & 6 & 0 & 29 & $9.86 \%$ \\
\hline
\end{tabular}


All the C. neoformans-positive patients were between the 26 to 45 age ranges. Direct examinations in India ink preparations of the 294 specimen types revealed polysaccharide capsules in 25 (8.5\%) samples: 20 CSFs, 5 urines and 0 in the blood samples. Upon culture, twenty nine (29) C. neoformans were identified: 23 from the CSFs and 6 from the urine. In 4 samples, culture was positive although India ink preparation was negative; three from CFS samples and one from urine. Twenty one out of $60(35 \%)$ patients and 8 out of $35(22.85 \%)$ of the category C and B respectively of the CDC classification System for HIV Infection yielded C. neoformans isolates. None of the CDC category A grew C. neoformans. Forty nine patients were on ART ( 3 from category A, 14 from category $\mathrm{B}$ and 32 from category $\mathrm{C}$ of $\mathrm{CDC}$ classification). Meanwhile, 56 (7 from category A, 21 from category $\mathrm{B}$, and 28 from category $\mathrm{C}$ of CDC classification) were not. Meningo-encephalitis symptoms were observed in $13(61.90 \%)$ patients with Cryptococcus positive CSFs. All these patients were also presenting visual impairment. None of the patients was undergoing antifungal therapy.

\section{Discussion}

Among the positive cases, 14 were males (66.66\%) and 7 females $(33.33 \%)$ indicating that male were more slightly involved than female. This may reflect the difference of exposure rather than difference in host susceptibility ${ }^{5,6}$. According to some authors, the reason for male predominance is not known although increased environmental exposure, hormonal inûuences and D or genetic predisposition of the patients have been postulated to be the contributing factors ${ }^{7,8}$. The age ranges in C. neoformans-positive patients (26-45 years) obtained in this study fit in with findings from previous studies. Cryptococcosis has been described in patients ranging from 20 and 49 years old, and this was not associated with any speciûc age group in a study in Malaysia. However, high incidence of cryptococcosis among patients of the similar age group has also been documented by investigators from many parts of the world ${ }^{9,10}$. It should be noted that, this age group is the most infected by HIV in Cameroon ${ }^{11}$. Cryptococcosis is known to be associated with Acquired Immunodeficiency Syndrome (AIDS), although it has been found in other types of immunocompromised patients and it sometimes causes disease in HIV-negative patients ${ }^{12}$.

A prevalence of $7.14 \%$ was observed in this study. Compared to the results from other parts of the world, the prevalence of cryptococcosis varies from place to place ${ }^{13,14}$. The number of sample positive in India ink preparation (8.5\%) is not equal to those positive in culture $(9.86 \%)$. According to Kisenge et $\mathrm{a}^{15}$ and Kumar et $\mathrm{al}^{7}$ India ink is positive in $60 \%$ and $70-90 \%$ of AIDS patients respectively. In spite of the difference observed in this study, the two methods are complementary, and therefore, both are recommended for an effective diagnosis of cryptococcosis. But, this observation emphasizes the problem of the sensitivity of culture and India ink test in routine clinical practice and the necessity for it to be completed by other tests such as Cryptococcus antigen detection in the samples. Among the 54 positive cases obtained both in CSF and urine, $43(44.32 \%)$ were from CSF while $11(10.47 \%)$ were from urine indicating that diagnosis of cryptococcosis is mainly based on cerebrospinal fluid (CSF). The CD 4 counts for patients varied between 31 and 304 lymphocytes $/ \mathrm{mm}^{3}$.

The CD4 count is the best indicator of immediate state of immunologic competence and also the strongest predictor of HIV-related complications in patients. The CDC categorization of HIV/AIDS is based on the lowest documented CD4 cell count and on previously diagnosed HIVrelated conditions ${ }^{16}$. According to the $\mathrm{CDC}$ clinical classification of HIV/AIDS, the patients were 60 , 35 and 10 in the C, B, and A categories respectively. All samples positive of $C$. neoformans were from patients that were not on ART. It has been shown that HIV-infected patients who are given combined antiretroviral therapy have a lower incidence of cryptococcosis, and the risk of cryptococcal disease is substantially reduced among persons receiving these treatments ${ }^{17}$. Antinori et al ${ }^{18}$ also observed that, with the introduction of HAART for HIV treatment in HIV patients, the incidence of cryptococcosis, along with other opportunistic infections, has decreased. The most frequent clinical manifestations involved the central nervous system and included headache and vomiting.

Visual impairment was also observed to be related with meningo-encephalitis symptoms. These results agree with some previous findings ${ }^{19,20}$. Fevers and headaches dominated the clinical features. This is similar to that described earlier ${ }^{21,22,23}$. The incidence of cryptococcosis in HIV patients, together with the amplitude of symptoms are lower than those obtained in a similar study in the same locality about twenty years back by Lohoue et $\mathrm{al}^{24}$. Despite the fact that in this study we work on a larger sample 
size compared to the one used by Lohoue et al (105 against 32 ), the decrease observed in the prevalence of cryptococcosis in HIV patients can be explained by the fact that recent advances in medicine have resulted in better patient management and prolonged life of the AIDS patients with cryptococcosis.

\section{Conclusion}

From this study, it is clear that cryptococcosis is rife in AIDS patients in Yaounde. To minimize the death toll, its routine check should be integrated in the management of AIDS patients particularly as the symptoms are not pathonomonic. We recommend that laboratory diagnosis of cryptococcal infections should be performed on HIV patients and accessibility to ARV therapy should be intensified as the risk of cryptococcal diseases is reduced among people receiving these treatments.

\section{References}

1. Speed B, Dunt D. Clinical and host differences between infections with the two varieties of Cryptococcus neoformans. Clin Infect Dis 1995, 21(1): 28-36.

2. Wallace RS, Meyer W, Wanke B, Costa SPSE, Trilles L, Nascimento JLM, et al. Primary endemic Cryptococcosis gattii by molecular type VGII in the state of Pará, Brazil. Mem Inst Oswaldo Cruz 2008, 103(8): 813-818.

3. Jain N, Wickes BL, Keller SM, Fu J, Casadevall A, Jain P, et al. Molecular Epidemiology of Clinical Cryptococcus neoformans Strains from India. J Clin Microbiol 2005, 43(11): 5733-5742.

4. Prado M, Da Silva MB, Laurenti R, Travassos L, Taborda C. Mortality due to systemic mycoses as a primary cause of death or in association with AIDS in Brazil: a review from 1996 to 2006. Mem Inst Oswaldo Cruz 2009, 104(3): 513521.

5. Lindenberg A, De SC, Chang MR, Paniago AMM, Lazéra M Dos S, Moncada PMF, et al. Clinical and epidemiological features of 123 cases of cryptococcosis in Mato Grosso do Sul, Brazil. Rev. Inst. Med. trop. S. Paulo 2008, 50(2): 75-78.

6. Prasad KN, Argawal J, Nag VL, Verma AK, Dixit AK, Ayyagari A. Cryptococcal infection in patients with clinically diagnosed meningitis in a tertiary care center. Neurol India 2003, 51(3): 364-366.

7. Kumar S, Wanchu A, Chakrabarti A, Sharma A, Bambery P, et al. Cryptococcal meningitis in HIV infected experience from a North Indian tertiary center. Neurol India 2008, 56:444-9.

8. Tay ST, Rohani MY, Soo Hoo TS, Hamimah H. Epidemiology of cryptococcosis in Malaysia. Mycoses 2009, 53: 509-514.

9. Tintelnot K, Lemmer K, Losert H, Schar G, Polak A. Follow-up of epidemiological data of cryptococcosis in Austria, Germany and Switzerland with special focus on the characterization of clinical isolates. Mycoses 2004, 47: 455-464.

10. Chen S, Sorrell T, Nimmo G, Speed B, Currie B, Ellis D, et al. Epidemiology and host and varietydependent characterization of infection due to Cryptococcus neoformans in Australia and New Zealand. Clin Infect Dis 2000, 31: 499-508.

11. Mbanya DN, Zebaze R, Minkoulou EM, Binam F, Koulla S, Obounou A. Clinical and epidemiologic trends in HIV/AIDS Patients in a hospital setting of Yaounde, Cameroon: a 6year perspective. Int J Infect Dis 2002, 6: 134 138.

12. Sasisopin K, Sirirat W, Roongnapa P, Somnuek S. Cryptococcosis in human immunodeûciency virus-negative patients. Int J Infect Dis 2006, 10:72-78.

13. Thakur R, Sarma S, Kushwaha S. Prevalence of HIV-associated cryptococcal meningitis and utility of microbiological determinants for its diagnosis in a tertiary care center. Indian J Path Microbiol 2008, 51(2): 212-214.

14. Taneji J, Mishra B, Bhargava A, Loomba P, Dogra $\mathrm{V}$, Thakur A. Cryptococcal meningitis in a tertiary care hospital. Jpn J Med Mycol 2009, 50: 95-99.

15. Kisenge PR, Hawkins A.T, Maro V P, Mchele JPD, Swai NS, Mueller A, Houpt ER. Low CD4 count plus coma predicts cryptococcal meningitis in Tanzania. BMC Infect Dis 2007, 7:39

16. Centers for Disease Control and Prevention. Revised classification system for HIV infection and expanded surveillance case definiton for AIDS among adolescents and adults. $M M W R$ (Morb Mortal Wkl Rep) 1992, 41(RR-17):1-19.

17. Park BJ, Wannemuehler KA, Marstonc BJ, Govender N, Pappas PG, Chiller TM. Estimation of the current global burden of cryptococcal meningitis among persons living with HIV/AIDS. AIDS 2009, 23(4):531-532.

18. Antinori S, Ridolfo A, Fasan M, Magni C, Galimberti L, Milazzo L, Sollima S, et al. AIDSassociated cryptococcosis: a comparison of 
epidemiology, clinical features and outcome in the pre- and post-HAART eras. Experience of a single centre in Italy. HIV Med 2009, 10: 611.

19. Ubonvan J, Somnuek S, Sasisopin K. Comparison of clinical features and survival between cryptococcosis in Human Immunodeficiency Virus (HIV)-positive and HIV-negative patients. Jpn J Infect Dis 2008, 61: 111-115.

20. Baradkar V, Mathur M, De A, Kumar S, Rathi M. Prevalence and clinical presentation of cryptococcal meningitis among HIV seropositive patients. Indian J Sex Transm Dis \& AIDS 2009, 30(1): 19-22.

21. Bogaerts J, Roubroy D, Taelman H, Kagame A, Aziz MA, Swinne D, et al: AIDS-associated cryptococcal meningitis in Rwanda (1983-1992). epidemiologic and diagnostic features. J Infect 1999, 39:32-37.

22. Gumbo T, Kadzirange G, Mielke J, Gangaidzo IT, Hakim JG. Cryptococcus neoformans meningoencephalitis in African children with acquired immunodeficiency syndrome. Pediatr Infect Dis J 2002, 21:54-56.

23. Pastagia M, Caplivski D. Disseminated cryptococcosis resulting in miscarriage in a woman without other immunocompromise: a case report. Int J Infect Dis 2010; 14: e441-e443.

24. Lohoue PJ, Same EA, Nomo OSA. Cryptococcose et syndrome de l'immunodéficience acquise à Yaoundé. Méd Mal Infect 1992, 22(1): 30-31. 